\title{
Alpha Power Inverted Kumaraswamy Distribution: Definition, Different Estimation Methods and Application
}

\author{
Kubra Bagci ${ }^{1}$, Necati Erdogan ${ }^{2}$, \\ Talha $\operatorname{Arslan}^{3 *}, \mathrm{H}$. Eray Celik ${ }^{4}$
}

${ }^{*}$ Corresponding author

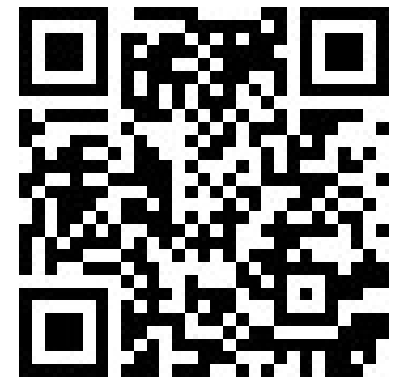

1. Department of Econometrics, Van Yüzüncüyıl University, 65080 Van, Turkey, kubrabagci@yyu.edu.tr

2. Department of Econometrics, Van Yüzüncüy1l University, 65080 Van, Turkey, nerdogan@yyu.edu.tr

3. Department of Econometrics, Van Yüzüncüyıl University, 65080 Van, Turkey, mstalhaarslan@yyu.edu.tr

4. Department of Econometrics, Van Yüzüncüy1l University, 65080 Van, Turkey, hecelik@yyu.edu.tr

\begin{abstract}
In this study, an alpha power inverted Kumaraswamy distribution having three shape parameters is obtained by applying the alpha power transformation to the inverted Kumaraswamy distribution. Then, its survival and hazard rate functions are expressed in closed forms. Some of its submodels and limiting cases are provided as well. Its parameters are estimated by using the maximum likelihood, maximum product of spacings, and least squares methods. A Monte-Carlo simulation study is conducted to show the performances of the considered estimation methods. An application to a real data set including values of breaking stress of carbon fibers is provided to illustrate an implementation of the proposed distribution and its modeling capability. The results show that alpha power inverted Kumaraswamy distribution can be an alternative to the its rivals.
\end{abstract}

Key Words: Alpha power transformation; Inverted Kumaraswamy distribution; Least squares; Maximum likelihood; Maximum product of spacings

Mathematical Subject Classification: 60E05, 62E15.

\section{Introduction}

Probability distribution functions are usually used for modeling data from various fields. Some of the probability distributions are identified with the relevant field of science. For example, the Kumaraswamy distribution introduced by Kumaraswamy (1980) has been generally known for its applications in the hydrology. After with Jones' (2009) detailed work on the Kumaraswamy distribution, it has become more familiar to statisticians. Recently, AL-Fattah et al. (2017) presented a transformation of the Kumaraswamy distribution, called as inverted Kumaraswamy (IKum) having a longer right tail when compared with the other well-known distributions. They stated that it allows more accurate predictions of rare events existing in the right tail of the distribution, i.e., long term reliability predictions. Later on, Abu-Moussa and El-Din (2018) obtained the maximum likelihood (ML) and Bayesian estimates for parameters of the IKum distribution under the progressive type-II censoring scheme. Aly and Abuelamayem (2020) derived the multivariate IKum distribution and used the ML and Bayesian methods to estimate its parameters. Hameed et al. (2020) conducted a study to estimate the stress strength reliability of a component under the IKum distribution. Recently, Bagci et al. (2021) used the IKum distribution to model the wind speed data and estimated its parameters by using the maximum product of spacings (MPS) and least squares (LS) methods.

A random variable $Z$ following the IKum distribution, i.e., $Z \sim \operatorname{IKum}(\beta, \lambda)$, has the probability density function (pdf) 


$$
f_{Z}(z ; \beta, \lambda)=\beta \lambda(1+z)^{-(\lambda+1)}\left[1-(1+z)^{-\lambda}\right]^{\beta-1} ; \quad z>0, \quad \beta>0, \quad \lambda>0
$$

and the cumulative distribution function (cdf)

$$
F_{Z}(z ; \beta, \lambda)=\left[1-(1+z)^{-\lambda}\right]^{\beta} ; \quad z>0, \quad \beta>0, \quad \lambda>0 .
$$

Here, $\beta$ and $\lambda$ are the shape parameters. Statistical properties of the IKum distribution are provided by AL-Fattah et al. (2017).

In recent years, number of studies have been carried out for extending the IKum distribution. For example, Iqbal et al. (2017) presented the generalized inverted Kumaraswamy (GIKw) distribution by using the power transformation as a more flexible model from the IKum distribution and its sub-models. Jamal et al. (2019) derived the GIKw generated (GIKw-G) family of distributions. Reyad et al. (2019) proposed the Topp-Leone generalized inverted Kumaraswamy distribution. Usman and ul Haq (2020) introduced the Marshall-Olkin extended inverted Kumaraswamy (MOEIK) distribution. Sherwani et al. (2021) used quadratic rank transmutation map to obtain the transmuted inverted Kumaraswamy distribution.

There are several methods for extending/generating statistical distributions; see Lee et al. (2013). These methods are generally used for making an existing distribution more flexible in terms of modeling capability. Recently, Mahdavi and Kundu (2017) used the alpha power transformation (APT) for generating a probability distribution. The APT method is conceptually based on adding a parameter to the distribution function to bring more flexibility to it. The APT method has some advantages of being easy applicability. Let $F(t)$ be a cdf and $f(t)$ be a pdf of a random variable $T$. The APT of $F(t)$ and $f(t)$ for $t \in \mathbb{R}$ are

$$
F_{A P T}(t)=\left\{\begin{array}{lll}
\frac{\alpha^{F_{T}(t)}-1}{\alpha-1} & ; \quad \alpha>0, \quad \alpha \neq 1 \\
F_{T}(t) & ; \quad \alpha=1
\end{array}\right.
$$

and

$$
f_{A P T}(t)= \begin{cases}\frac{\ln (\alpha)}{\alpha-1} f_{T}(t) \alpha^{F_{T}(t)} & ; \quad \alpha>0, \quad \alpha \neq 1 \\ f_{T}(t) & ; \quad \alpha=1,\end{cases}
$$

respectively. See Mahdavi and Kundu (2017) for the details.

Notice that the APT family of distributions is reparemetrized version of the "exp-G" family of distributions introduced by Barreto-Souza and Simas (2014) and the equivalent "truncated-exponential skew-symmetric" family of distributions obtained by Nadarajah et al. (2014) as stated in Jones (2018). Beside with keeping mind this fact, for the purpose of being parallel to the recent literature, the APT terminology will be used in the rest of this study.

In the literature, the APT method has been applied to many different distributions. For example, Dey et al. (2017) obtained the alpha power generalized Exponential distribution. Nassar et al. (2017) introduced the alpha power Weibull distribution. Hassan et al. (2018) derived the alpha power extended Exponential distribution. Unal et al. (2018) obtained the alpha power inverted Exponential distribution. Ramadan and Magdy (2018) and Basheer (2019) proposed the alpha power inverse Weibull distribution. Dey et al. (2019a, 2019b) introduced the alpha power Lindley and alpha power inverse Lindley distributions. Hassan et al. (2019) derived the alpha power power-Lindley distribution. Ihtisham et al. (2019) proposed the alpha power Pareto distribution. Erdogan et al. (2021) obtained the alpha power Maxwell distribution.

This study presents the following contributions to the related literature. (i) An alpha power inverted Kumaraswamy (APIK) distribution is introduced as an alternative to the IKum and some of its extended/generalized versions such as the MOEIK and GIKw-W distributions. (ii) Several submodels and limiting cases of the APIK distribution, to the best of the Authors' knowledge, some of them have not been proposed yet, are obtained as well. (iii) The ML, MPS and LS methods are used to estimate the unknown parameters of the APIK distribution. The performances of the considered estimation methods are compared via a Monte-Carlo simulation study. Note that the earlier version of this study was presented at the 4th International Conference on Computational Mathematics and Engineering Sciences (CMES-2019); see Bagci et al. (2019). 
The rest of this study is organized as follows. In Section 2, the pdf, cdf, and some statistical inferences of the APIK distribution are obtained. In Section 3, sub-models and limiting cases of the APIK distribution, and also distributions obtained by applying different variable transformations are derived. Afterward, in Section 4, the ML, MPS, and LS estimation for parameters of the APIK distribution are given, and then the Monte-Carlo simulation results for comparing the performances of these methods are provided. In Section 5, a real data set is modeled by using the APIK distribution and some of its strong alternatives to compare their modeling performances with each other. Finally, the last section reserved for some concluding remarks.

\section{The APIK Distribution}

In this section, the APIK distribution is obtained by applying the APT method to the IKum distribution.

Definition 2.1. A random variable $X$ following the APIK distribution, i.e., $X \sim A P I K(\alpha, \beta, \lambda)$, has the cdf

$$
F_{X}(x, \alpha, \beta, \lambda)=\left\{\begin{array}{lllll}
\frac{\alpha^{\left[1-(1+x)^{-\lambda}\right]^{\beta}}-1}{\alpha-1} & ; \quad x>0, \quad \beta>0, & \lambda>0, \quad \alpha>0, \quad \alpha \neq 1 \\
F_{Z}(x ; \beta, \lambda) & ; \quad x>0, \quad \beta>0, \quad \lambda>0, \quad \alpha=1
\end{array}\right.
$$

and the pdf

$$
f_{X}(x ; \alpha, \beta, \lambda)=\left\{\begin{array}{llll}
\frac{\ln \alpha}{\alpha-1} \beta \lambda(1+x)^{-(\lambda+1)}\left[1-(1+x)^{-\lambda}\right]^{\beta-1} \alpha^{\left[1-(1+x)^{-\lambda}\right]^{\beta}} & ; \quad x>0, \quad \beta>0, \quad \lambda>0, \quad \alpha>0, \quad \alpha \neq 1 \\
f_{Z}(x ; \beta, \lambda) & ; \quad x>0, \quad \beta>0, \quad \lambda>0, \quad \alpha=1 .
\end{array}\right.
$$

Definition 2.2. Let $X \sim A P I K(\alpha, \beta, \lambda)$, then it has the survival function

$$
S_{X}(x, \alpha, \lambda, \beta)=\left\{\begin{array}{llll}
\frac{\alpha}{\alpha-1}\left[1-\alpha^{\left[1-(1+x)^{-\lambda}\right]^{\beta}-1}\right] & ; \quad x>0, \quad \beta>0, \quad \lambda>0, \quad \alpha>0, \quad \alpha \neq 1 \\
1-F_{Z}(x ; \beta, \lambda) & ; \quad x>0, \quad \beta>0, \quad \lambda>0, \quad \alpha=1
\end{array}\right.
$$

and the hazard rate function (hrf)

$$
H_{X}(x ; \alpha, \beta, \lambda)=\left\{\begin{array}{lll}
(\ln \alpha)\left[\beta \lambda(1+x)^{-(\lambda+1)}\left(1-(1+x)^{-\lambda}\right)^{\beta-1}\right] \frac{\alpha^{\left[1-(1+x)^{-\lambda}\right]^{\beta}-1}}{1-\alpha^{\left[1-(1+x)^{-\lambda}\right]^{\beta}-1}} & ; \quad x>0, \quad \beta>0, \quad \lambda>0, \quad \alpha>0, \quad \alpha \neq 1 \\
\frac{f_{Z}(x ; \beta, \lambda)}{1-F_{Z}(x ; \beta, \lambda)} & ; \quad x>0, \quad \beta>0, \quad \lambda>0, \quad \alpha=1 .
\end{array}\right.
$$

The pdf, cdf and hrf of the APIK distribution, given in (3), (4) and (5), respectively, are plotted for different parameters settings in Figure 1(a), Figure 1(b), and Figure 1(c), respectively.

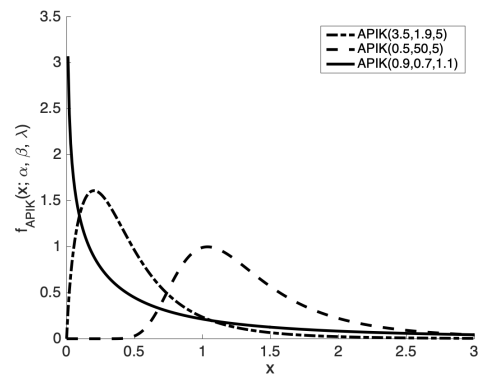

(a)

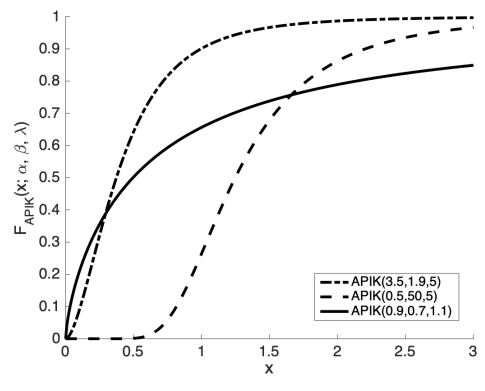

(b)

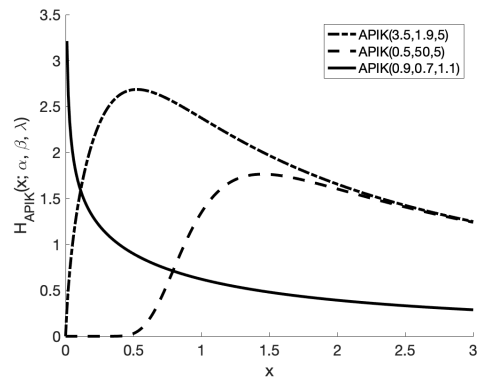

(c)

Figure 1: The pdf (a), cdf (b), and hrf (c) plots of the APIK distribution for different values of its parameters.

It is clear from the Figure 1(a) and Figure 1(c) that the pdf and hrf of the APIK distribution can be monotone decrease or monotone increase-decrease for the different parameter settings, respectively. 
Proposition 2.1. $\lim _{\alpha \rightarrow 1} f_{X}(x ; \alpha, \beta, \lambda)=f_{Z}(x ; \beta, \lambda)$.

Proof. It follows from due to the fact that $\lim _{\alpha \rightarrow 1} \frac{\ln \alpha}{\alpha-1}=1$ and $\lim _{\alpha \rightarrow 1} \alpha^{F_{Z}(x ; \beta, \lambda)}=1$. For the cases exp-G family of distributions and alpha power Maxwell distribution, see Barreto-Souza and Simas (2014) and Erdogan et al. (2021), respectively.

Due to the fact given in Proposition 2.1, Definition 2.3 is obtained only for the APIK distribution, i.e., $\alpha \neq 1$, for the sake of brevity.

Definition 2.3. The quantile function of the APIK distribution, i.e., inverse of the cdf of the APIK distribution, is

$$
Q(p ; \alpha, \beta, \lambda)=\left[1-\left(\frac{\ln (1+(\alpha-1) p)}{\ln \alpha}\right)^{\frac{1}{\beta}}-1 ; \quad 0<p<1, \quad \beta>0, \quad \lambda>0, \quad \alpha>0, \quad \alpha \neq 1 .\right.
$$

Hence, the median of the APIK distribution is

$$
Q(0.5 ; \alpha, \beta, \lambda)=\left[1-\left(\frac{\ln (1+(\alpha-1) 0.5)}{\ln \alpha}\right)^{\frac{1}{\beta}}\right]^{-\frac{1}{\lambda}}-1 ; \quad \beta>0, \quad \lambda>0, \quad \alpha>0, \quad \alpha \neq 1 .
$$

\section{Related Distributions}

The APIK distribution includes some well-known distribution as a sub-model. It also converges to the some other distributions as a limiting case under different variable transformations. Here, referred distributions are given briefly.

It should be mentioned that following distributions are obtained by using the variable transformations and some limiting cases which were already defined by AL-Fattah et al. (2017).

\subsection{Sub-models of the APIK distribution}

The sub-models of the APIK distribution are obtained for different parameters settings as given below.

(i) When $\beta=1$, it reduces to the alpha power Lomax $(\alpha, \lambda, 1)$ distribution:

$$
f_{X}(x ; \alpha, 1, \lambda)=\left\{\begin{array}{lll}
\frac{\ln \alpha}{\alpha-1} \lambda(1+x)^{-(\lambda+1)} \alpha^{1-(1+x)^{-\lambda}} & ; \quad x>0, \quad \lambda>0, \quad \alpha>0, \quad \alpha \neq 1 \\
\lambda(1+x)^{-(\lambda+1)} & ; \quad x>0, \quad \lambda>0, \quad \alpha=1
\end{array}\right.
$$

(ii) When $\lambda=1$, it reduces to the alpha power Beta Type II $(\alpha, \beta, 1)$ distribution:

$$
f_{X}(x ; \alpha, \beta, 1)=\left\{\begin{array}{lll}
\ln \alpha \\
\frac{\ln }{\alpha-1} x^{\beta-1}(1+x)^{-(\beta+1)} \alpha\left(\frac{x}{1+x}\right)^{\beta} & ; \quad x>0, \quad \beta>0, \quad \alpha>0, \quad \alpha \neq 1 \\
\beta x^{\beta-1}(1+x)^{-(\beta+1)} & ; \quad x>0, \quad \beta>0, \quad \alpha=1
\end{array}\right.
$$

(iii) When $\beta=1$ and $\lambda=1$, it reduces to the alpha power $\log$-logistic $(1,1, \alpha)$ distribution:

$$
f_{X}(x ; \alpha, 1,1)=\left\{\begin{array}{lll}
\ln \alpha \\
\frac{\ln }{\alpha-1}(1+x)^{-2} \alpha\left(\frac{x}{1+x}\right) & ; \quad x>0, \quad \alpha>0, \quad \alpha \neq 1 \\
(1+x)^{-2} & ; \quad x>0, \quad \alpha=1
\end{array}\right.
$$

Note that the alpha power Log-Logistic and the alpha power Lomax distributions are introduced by Aldahlan (2020) and Bulut et al. (2021), respectively. Also, notice that the alpha power Lomax distribution is a submodel of the Marshall-Olkin alpha power Lomax distribution introduced by Almongy et al. (2021). 


\subsection{Transformations}

A random variable $Y$ has the following pdf and also its submodels for the certain parameter settings.

(i) $Y=[\ln (1+X)]^{\frac{1}{\theta}}$ follows the alpha power exponentiated Weibull $(\alpha, \theta, \lambda, \beta)$ distribution, see Mead et al. (2019), having the pdf

$$
f_{Y}(y ; \alpha, \beta, \lambda, \theta)= \begin{cases}\frac{\ln \alpha}{\alpha-1} \beta \lambda \theta y^{\theta-1} e^{-\lambda y^{\theta}}\left(1-e^{-\lambda y^{\theta}}\right)^{\beta-1} \alpha^{\left(1-e^{-\lambda y^{\theta}}\right)^{\beta}} & ; \quad \alpha \neq 1 \\ \beta \lambda \theta y^{\theta-1} e^{-\lambda y^{\theta}}\left(1-e^{-\lambda y^{\theta}}\right)^{\beta-1} & ; \quad \alpha=1\end{cases}
$$

where $y>0, \quad \alpha>0, \quad \beta>0, \quad \lambda>0, \quad$ and $\theta>0$.

Sub-models

\begin{tabular}{cl}
\hline$\theta=2$ & Alpha Power generalized Rayleigh $(\alpha, \beta, \lambda)$; see Biçer (2019) \\
$\theta=1$ & Alpha Power generalized Exponential $(\alpha, \beta, \lambda)$; see Dey et al. (2017) \\
$\beta=1$ & Alpha Power Weibull $(\alpha, \lambda, \theta) ;$ see Nassar et al. (2017) \\
$\theta=2$ & Alpha Power Burr Type $\mathrm{X}(\alpha, \lambda, \beta)$ \\
$\beta=1$ and $\theta=2$ & Alpha Power Rayleigh $(\alpha, \lambda)$ \\
$\theta=1$ and $\beta=1$ & Alpha Power Exponential $(\alpha, \lambda) ;$ see Mahdavi and Kundu (2017) \\
\hline
\end{tabular}

(ii) $Y=\lambda \sigma \ln (1+X)+\mu$ follows the alpha power generalized shifted Exponential $(\alpha, \beta, \mu, \sigma)$ distribution having the pdf

$$
f_{Y}(y ; \alpha, \beta, \mu, \sigma)= \begin{cases}\frac{\ln \alpha}{\alpha-1} \frac{\beta}{\sigma} e^{-\left(\frac{y-\mu}{\sigma}\right)}\left[1-e^{-\left(\frac{y-\mu}{\sigma}\right)}\right]^{\beta-1} \alpha\left[1-e^{-\left(\frac{y-\mu}{\sigma}\right)}\right]^{\beta} & ; \quad \alpha \neq 1 \\ \frac{\beta}{\sigma} e^{-\left(\frac{y-\mu}{\sigma}\right)}\left[1-e^{-\left(\frac{y-\mu}{\sigma}\right)}\right]^{\beta-1} & ; \quad \alpha=1\end{cases}
$$

where $\mu<y<\infty, \quad \alpha>0, \quad \beta>0, \quad$ and $\quad \sigma>0$.

Sub-models

\begin{tabular}{ll}
\hline$\beta=1$ & Alpha power shifted Exponential $(\alpha, \mu, \sigma)$ \\
$\beta=1$ and $\mu=0$ & Alpha power Exponential $(\alpha, \sigma)$ \\
\hline
\end{tabular}

(iii) $Y=\sigma X^{\frac{1}{\theta}}$ follows the alpha power exponentiated Burr Type XII $(\alpha, \beta, \lambda, \theta, \sigma)$ distribution having the pdf

$$
f_{Y}(y ; \alpha, \beta, \lambda, \theta, \sigma)=\left\{\begin{array}{lr}
\frac{\ln \alpha}{\alpha-1} \frac{\lambda \beta \theta}{\sigma}\left(\frac{y}{\sigma}\right)^{\theta-1}\left[1+\left(\frac{y}{\sigma}\right)^{\theta}\right]^{-(\lambda+1)}\left[1-\left(1+\left(\frac{y}{\sigma}\right)^{\theta}\right)^{-\lambda}\right]^{\beta-1} \alpha^{\left[1-\left(1+\left(\frac{y}{\sigma}\right)^{\theta}\right)^{-\lambda}\right]^{\beta}} & ; \quad \alpha \neq 1 \\
\frac{\lambda \beta \theta}{\sigma}\left(\frac{y}{\sigma}\right)^{\theta-1}\left[1+\left(\frac{y}{\sigma}\right)^{\theta}\right]^{-(\lambda+1)}\left[1-\left(1+\left(\frac{y}{\sigma}\right)^{\theta}\right)^{-\lambda}\right]^{\beta-1} & \alpha=1
\end{array}\right.
$$

where $y>0, \quad \alpha>0, \quad \beta>0, \quad \lambda>0, \quad \theta>0, \quad$ and $\quad \sigma>0$.

\section{Sub-models}

\begin{tabular}{ll}
\hline$\beta=1$ & Alpha power Burr Type XII $(\alpha, \lambda, \theta, \sigma)$ \\
$\theta=1$ and $\sigma=1$ & Alpha power generalized Lomax $(\alpha, \beta, \lambda)$ \\
$\beta=1$ & Alpha power generalized Beta Type II $(\alpha, 1, \lambda, \theta, \sigma)$ \\
$\beta=1, \theta=1$, and $\lambda=\sigma$ & Alpha power F-distribution $(\alpha, 2,2 \lambda)$ \\
\hline
\end{tabular}




\subsection{Limiting Distributions}

The random variable $Y$ has the following pdf for the certain parameter settings.

(i) $Y=\beta^{-\frac{1}{\lambda}}(1+X)$ and $\beta \rightarrow \infty$, then $Y$ follows the alpha power inverse Weibull $(\alpha, \lambda, 1)$ distribution, see Ramadan and Magdy (2018) and Basheer (2019), having the pdf

$$
f(y ; \alpha, \lambda)=\left\{\begin{array}{lll}
\frac{\ln \alpha}{\alpha-1} \lambda y^{-(\lambda+1)} e^{-y^{-\lambda}} \alpha^{e^{-y^{-\lambda}}} & ; \quad y>0, \quad \lambda>0, \quad \alpha>0, \quad \alpha \neq 1 \\
\lambda y^{-(\lambda+1)} e^{-y^{-\lambda}} & ; \quad y>0, \quad \lambda>0, \quad \alpha=1
\end{array}\right.
$$

(ii) $Y=\lambda\left[1-(1+X)^{-1}\right]$ and $\lambda \rightarrow \infty$ then $Y$ follows the alpha power generalized Exponential $(\alpha, \beta, 1)$ distribution, see Dey et al. (2017), having the pdf

$$
f(y ; \alpha, \beta)=\left\{\begin{array}{llll}
\frac{\ln \alpha}{\alpha-1} \beta e^{-y}\left(1-e^{-y}\right)^{\beta-1} \alpha^{\left(1-e^{-y}\right)^{\beta}} & ; \quad y>0, \quad \beta>0, \quad \alpha>0, \quad \alpha \neq 1 \\
\beta e^{-y}\left(1-e^{-y}\right)^{\beta-1} & ; \quad y>0, \quad \beta>0, \quad \alpha=1
\end{array}\right.
$$

(iii) $Y=\lambda\left[1-\beta^{\frac{1}{\lambda}}(1+X)^{-1}\right]$ and $\beta, \lambda \rightarrow \infty$, then $Y$ follows the alpha power standard Gumbel distribution having the pdf

$$
f(y ; \alpha)= \begin{cases}\frac{\ln \alpha}{\alpha-1} e^{-y} e^{-e^{-y}} \alpha^{e^{-e^{-y}}} & ; \quad y \in \mathbb{R}, \quad \alpha>0, \quad \alpha \neq 1 \\ e^{-y} e^{-e^{-y}} & ; \quad y \in \mathbb{R}, \quad \alpha=1\end{cases}
$$

Note that

$$
\begin{gathered}
\lim _{\lambda \rightarrow \infty}\left(1-\frac{y}{\lambda}\right)^{\lambda-1}=e^{-y}, \lim _{\lambda \rightarrow \infty}\left[1-\left(1-\frac{y}{\lambda}\right)^{\lambda}\right]^{\beta-1}=\left(1-e^{-y}\right)^{\beta-1}, \lim _{\beta \rightarrow \infty}\left[1-\beta^{-1} y^{-\lambda}\right]^{\beta-1}=e^{-y^{-\lambda}, \quad \text { and }} \\
\lim _{\beta \rightarrow \infty}\left\{\lim _{\lambda \rightarrow \infty}\left[1-\beta^{-1}\left(1-\frac{y}{\lambda}\right)^{\lambda}\right]^{\beta-1}\right\}=e^{-e^{-y}} .
\end{gathered}
$$

\section{Parameter estimation}

In this section, the ML, MPS and LS estimation methods are used for estimating the parameters $\alpha, \beta$ and $\lambda$ of the APIK distribution.

\subsection{ML estimation}

Let $x_{1}, x_{2}, \ldots, x_{n}$ be a random sample from the APIK distribution. Then, the $\log$-likelihood $(\ln L)$ function of it is

$$
\begin{aligned}
\ln L(\alpha, \beta, \lambda ; \underset{\sim}{x}) & =n \ln (\ln \alpha)-n \ln (\alpha-1)+n \ln (\beta)+n \ln (\lambda)-(\lambda+1) \sum_{i=1}^{n} \ln \left(1+x_{i}\right) \\
& +(\beta-1) \sum_{i=1}^{n} \ln \left[1-\left(1+x_{i}\right)^{-\lambda}\right]+(\ln \alpha) \sum_{i=1}^{n}\left[1-\left(1+x_{i}\right)^{-\lambda}\right]^{\beta}
\end{aligned}
$$

The likelihood equations

$$
\begin{gathered}
\frac{\partial \ln L}{\partial \alpha}=\frac{n}{\alpha \ln \alpha}-\frac{n}{\alpha-1}+\frac{1}{\alpha} \sum_{i=1}^{n}\left[1-\left(1+x_{i}\right)^{-\lambda}\right]^{\beta}=0 \\
\frac{\partial \ln L}{\partial \beta}=\frac{n}{\beta}+\sum_{i=1}^{n} \ln \left[1-\left(1+x_{i}\right)^{-\lambda}\right]+(\ln \alpha) \sum_{i=1}^{n}\left\{\left[1-\left(1+x_{i}\right)^{-\lambda}\right]^{\beta} \ln \left[1-\left(1+x_{i}\right)^{-\lambda}\right]\right\}=0
\end{gathered}
$$

and 


$$
\frac{\partial \ln L}{\partial \lambda}=\frac{n}{\lambda}-\sum_{i=1}^{n} \ln \left(1+x_{i}\right)+(\beta-1) \sum_{i=1}^{n}\left[\frac{\ln (1+x)}{(1+x)^{\lambda}-1}\right]+\beta(\ln \alpha) \sum_{i=1}^{n} \frac{\ln \left(1+x_{i}\right)}{\left(1+x_{i}\right)^{\lambda}}\left[1-\left(1+x_{i}\right)^{-\lambda}\right]^{\beta-1}=0
$$

are obtained by taking partial derivatives of the $\ln L$ function given in (6) with respect to the parameters $\alpha$, $\beta$ and $\lambda$, and equating them zero. The ML estimates of the parameters $\alpha, \beta$ and $\lambda$ are obtained by solving the likelihood equations (7) - (9), simultaneously.

\subsection{MPS estimation}

The MPS method is based on maximizing a geometric mean of spacing. Therefore, the MPS estimates of $\alpha, \beta$ and $\lambda$ of the APIK distribution are the points in which the objective function

$$
D(\alpha, \beta, \lambda ; \underset{\sim}{x})=\left(\frac{1}{n+1}\right) \sum_{i=0}^{n} \ln \left\{\left[\frac{\alpha^{\left[1-\left(1+x_{(i+1)}\right)^{-\lambda}\right]^{\beta}}-1}{\alpha-1}\right]-\left[\frac{\alpha^{\left[1-\left(1+x_{(i)}\right)^{-\lambda}\right]^{\beta}}-1}{\alpha-1}\right]\right\}
$$

attains its maximum. Here, $x_{(\cdot)}$ denotes ordered observation, i.e., $x_{(1)} \leq x_{(2)} \leq \cdots \leq x_{(n-1)} \leq x_{(n)}$. Note that $x_{0}=0$ and $x_{(n+1)}=\infty$ since the support for $F_{X}(x)$ is the positive real line. Therefore, $F_{X}\left(x_{0} ; \alpha, \beta, \lambda\right) \equiv 0$ and $F_{X}\left(x_{n+1} ; \alpha, \beta, \lambda\right) \equiv 1$. See Arslan and Oncel (2017), Volovskiy and Kamps (2020), and references given them for further information.

\subsection{LS estimation}

In the LS method, it is aimed to minimize a sum of squares of the differences between theoretical and expected quantiles with respect to the parameters of interest, i.e., $\alpha, \beta$ and $\lambda$. Therefore, the LS estimates of the unknown parameters $\alpha, \beta$ and $\lambda$ of the APIK distribution are the points in which the objective function

$$
S(\alpha, \beta, \underset{\sim}{\lambda} \underset{\sim}{x})=\frac{1}{n} \sum_{i=1}^{n}\left[\frac{\alpha^{\left[1-\left(1+x_{(i)}\right)^{-\lambda}\right]^{\beta}}-1}{\alpha-1}-\left(\frac{i}{n+1}\right)\right]^{2}
$$

attains its minimum; see Swain et al. (1988) for further information.

Note that optimization tools "fminsearch", "fminunc" or "ga" which are available in software MATLAB2015b can be used to find the ML, MPS, and LS estimates of the parameters $\alpha, \beta$, and $\lambda$. See also Arslan et al. (2017), Nassar et al. (2020) and Almongy et al. (2021) in the context of the MPS and LS estimation for parameters of the generalized Lindley and power Lindley, alpha power Exponential, and Marshall-Olkin alpha power Lomax distributions, respectively.

\subsection{Monte-Carlo simulation}

In this subsection, the Monte-Carlo simulation is conducted to compare performances of the ML, MPS, and LS methods in estimating the parameters $\alpha, \beta$ and $\lambda$ of the APIK distribution. The bias, variance and mean squared eror (MSE) criteria are used in the comparisons. The simulated values of the MSE criterion are calculated by using the equalities

$$
M S E(\hat{\alpha})=E(\hat{\alpha}-\alpha)^{2}, \quad M S E(\hat{\beta})=E(\hat{\beta}-\beta)^{2} \quad \text { and } \quad M S E(\hat{\lambda})=E(\hat{\lambda}-\lambda)^{2} .
$$

The joint efficiency criterion, i.e., Def, is also used for the parameters $\alpha, \beta$ and $\lambda$. The simulated values of the Def are calculated by using equality

$$
\operatorname{Def}(\hat{\alpha}, \hat{\beta}, \hat{\lambda})=M S E(\hat{\alpha})+M S E(\hat{\beta})+M S E(\hat{\lambda})
$$

see Akgül et al. (2016) and references therein.

The simulated values of the bias, variance, and MSE criteria are calculated for 1000 runs in different sample sizes $n=50,100$, and $n=300$ with the certain parameters settings. The simulation outcomes are given in Table 1 and 
summarized as follows.

i. When $\alpha=0.5, \beta=0.9, \lambda=2.5$ :

The MPS method gives the smallest bias values for $\alpha$ in all sample sizes. The LS method results larger bias values than the ML. In terms of the MSE criterion, the LS method produces the smallest values.

For the $\beta$, the ML, MPS, and LS methods have small biases for all sample sizes, however the ML method has the smallest and the MPS has the largest bias values for $n=100$. In terms of the MSE criterion, the MPS method gives the smallest values for $n=50$ and $n=100$.

Concerning the $\lambda$, the ML method results the smallest bias values for $n=100$ and $n=300$, however the LS produces the largest bias values. In terms of the MSE criterion, the MPS method gives the smallest values for $n=50$ and $n=100$.

Overall, the MPS method is the best for $n=50$ and 100 when the Def criterion taken into account.

ii. When $\alpha=0.9, \beta=1.5, \lambda=0.5$ :

The ML method gives the smallest bias values for $\alpha$ in all sample sizes. The LS method results the smallest variance values for all sample sizes. In terms of the MSE criterion, the LS method produces the smallest values.

For the $\beta$, the ML, MPS, and LS methods have the small biases for all sample sizes. The MPS method has the smallest variance values for all sample sizes. In terms of the MSE criterion, the MPS method gives the smallest values for $n=50$ and 100 .

Concerning the $\lambda$, the ML, MPS, and LS methods give small bias values, and the MPS method produces the smallest variance values for all sample sizes. In terms of the MSE criterion, the MPS method gives the smallest values for all sample sizes.

Overall, the LS method is the best for all sample sizes when the Def criterion taken into account.

iii. When $\alpha=1.1, \beta=0.5, \lambda=2.5$ :

The ML, MPS, and LS methods give large bias values for $\alpha$ in all sample sizes. However, the LS method produces the smallest MSE values for all sample sizes.

Concerning the $\beta$, the ML, MPS, and LS methods result small bias values for $n=50$ and 100 while the MPS method gives the smallest variance values for the corresponding sample sizes. When the MSE criterion is taken into account, the MPS method is preferable to the ML and LS since it produces the smallest MSE values for all sample sizes.

For the $\lambda$, the MPS method gives the largest bias values for $n=100$ and $n=300$, however it has the smallest variance values for all sample sizes. In terms of the MSE criterion, the MPS method is one step ahead of the ML and LS since it produces the smallest MSE values for all sample sizes.

Overall, the MPS, LS and ML methods are preferable for $n=50, n=100$, and $n=300$, respectively, when the Def criterion taken into account.

iv. When $\alpha=2.0, \beta=1.5, \lambda=1.1$ :

The ML method produces the smallest bias values for all sample sizes. The MPS method gives larger bias values than the LS, except $n=300$. In terms of the MSE criterion, the LS method is preferable over the ML and MPS.

Concerning the $\beta$, the LS method results the smallest bias values while the ML and MPS methods have also small bias for $n=100$ and $n=300$. When the MSE criterion is taken into account, the MPS shows better performance than the ML and LS since it produces the smallest MSE values for all sample sizes.

For the $\lambda$, the ML, MPS, and LS methods produce small bias values for all sample sizes. However, the MPS method gives the smallest variance and the MSE values for all sample sizes.

Overall, the MPS method is one step ahead of the ML and LS since it produces the smallest Def values for all sample sizes. 
Table 1: The simulated bias, variance, and MSE values of the $\hat{\alpha}, \hat{\beta}$ and $\hat{\lambda}$ for the ML, MPS, and LS methods. $\alpha=0.5, \beta=0.9, \lambda=2.5$

\begin{tabular}{|c|c|c|c|c|c|c|c|c|c|c|c|}
\hline & & \multicolumn{3}{|c|}{$\hat{\alpha}$} & \multicolumn{3}{|c|}{$\hat{\beta}$} & \multicolumn{3}{|c|}{$\hat{\lambda}$} & \multirow[b]{2}{*}{ Def } \\
\hline & & Bias & Variance & MSE & Bias & Variance & MSE & Bias & Variance & MSE & \\
\hline \multirow{3}{*}{$\mathrm{n}=50$} & ML & 0.1156 & 0.1037 & 0.1171 & 0.0431 & 0.0318 & 0.0337 & 0.2141 & 0.4621 & 0.5080 & 0.6587 \\
\hline & MPS & 0.0836 & 0.1096 & 0.1166 & -0.0405 & 0.0252 & 0.0269 & -0.1371 & 0.3918 & 0.4106 & 0.5541 \\
\hline & LS & 0.1537 & 0.0735 & 0.0971 & -0.0051 & 0.0369 & 0.0369 & 0.1432 & 0.4848 & 0.5053 & 0.6394 \\
\hline \multirow{3}{*}{$n=100$} & ML & 0.1056 & 0.0989 & 0.1101 & 0.0110 & 0.0149 & 0.0150 & 0.0795 & 0.2491 & 0.2554 & 0.3805 \\
\hline & MPS & 0.0807 & 0.1015 & 0.1080 & -0.0348 & 0.0130 & 0.0142 & -0.1264 & 0.2288 & 0.2447 & 0.3670 \\
\hline & LS & 0.1573 & 0.0611 & 0.0858 & -0.0148 & 0.0192 & 0.0194 & 0.1276 & 0.2698 & 0.2861 & 0.3913 \\
\hline \multirow{3}{*}{$n=300$} & ML & 0.0841 & 0.0723 & 0.0794 & -0.0024 & 0.0061 & 0.0061 & 0.0361 & 0.1118 & 0.1131 & 0.1986 \\
\hline & MPS & 0.0654 & 0.0724 & 0.0767 & -0.0189 & 0.0058 & 0.0062 & -0.0543 & 0.1102 & 0.1131 & 0.1959 \\
\hline & LS & 0.1504 & 0.0420 & 0.0646 & -0.0192 & 0.0064 & 0.0068 & 0.1340 & 0.1108 & 0.1288 & 0.2001 \\
\hline
\end{tabular}

$\alpha=0.9, \beta=1.5, \lambda=0.5$

\begin{tabular}{|c|c|c|c|c|c|c|c|c|c|c|c|}
\hline & \multicolumn{3}{|c|}{$\hat{\alpha}$} & \multicolumn{3}{|c|}{$\hat{\beta}$} & \multicolumn{3}{|c|}{$\hat{\lambda}$} & \multirow[b]{2}{*}{ Def } \\
\hline & & Bias & Variance & MSE & Bias & Variance & MSE & Bias & Variance & MSE & \\
\hline \multirow{3}{*}{$\mathrm{n}=50$} & ML & 0.2533 & 0.3252 & 0.3894 & 0.0936 & 0.1330 & 0.1418 & 0.0318 & 0.0071 & 0.0081 & 0.5393 \\
\hline & MPS & 0.3659 & 0.3165 & 0.4504 & -0.0793 & 0.1067 & 0.1130 & 0.0018 & 0.0043 & 0.0043 & 0.5677 \\
\hline & LS & 0.3872 & 0.1992 & 0.3491 & 0.0086 & 0.1476 & 0.1477 & 0.0306 & 0.0082 & 0.0092 & 0.5060 \\
\hline \multirow{3}{*}{$\mathrm{n}=100$} & ML & 0.1908 & 0.2853 & 0.3218 & 0.0458 & 0.0652 & 0.0673 & 0.0179 & 0.0043 & 0.0046 & 0.3937 \\
\hline & MPS & 0.2891 & 0.2868 & 0.3704 & -0.0601 & 0.0582 & 0.0618 & 0.0030 & 0.0032 & 0.0032 & 0.4355 \\
\hline & LS & 0.3039 & 0.1733 & 0.2656 & -0.0183 & 0.0800 & 0.0803 & 0.0214 & 0.0045 & 0.0050 & 0.3509 \\
\hline \multirow{3}{*}{$\mathrm{n}=300$} & ML & 0.1532 & 0.2186 & 0.2421 & -0.0043 & 0.0242 & 0.0242 & 0.0053 & 0.0019 & 0.0020 & 0.2682 \\
\hline & MPS & 0.2222 & 0.2259 & 0.2753 & -0.0514 & 0.0238 & 0.0265 & 0.0017 & 0.0017 & 0.0017 & 0.3035 \\
\hline & LS & 0.3037 & 0.1306 & 0.2228 & -0.0427 & 0.0281 & 0.0299 & 0.0199 & 0.0020 & 0.0024 & 0.2552 \\
\hline
\end{tabular}

$\alpha=1.1, \beta=0.5, \lambda=2.5$

\begin{tabular}{|c|c|c|c|c|c|c|c|c|c|c|c|}
\hline & \multicolumn{3}{|c|}{$\hat{\alpha}$} & \multicolumn{3}{|c|}{$\hat{\beta}$} & \multicolumn{3}{|c|}{$\hat{\lambda}$} & \multirow[b]{2}{*}{ Def } \\
\hline & & Bias & Variance & MSE & Bias & Variance & MSE & Bias & Variance & MSE & \\
\hline \multirow{3}{*}{$\mathrm{n}=50$} & ML & -0.1549 & 0.2923 & 0.3163 & 0.0828 & 0.0043 & 0.0112 & 0.1755 & 0.4458 & 0.4766 & 0.8042 \\
\hline & MPS & -0.2819 & 0.2140 & 0.2935 & 0.0671 & 0.0022 & 0.0067 & -0.1362 & 0.3541 & 0.3726 & 0.6728 \\
\hline & LS & -0.2331 & 0.2168 & 0.2711 & 0.0774 & 0.0035 & 0.0095 & 0.1143 & 0.5159 & 0.5290 & 0.8096 \\
\hline \multirow{3}{*}{$\mathrm{n}=100$} & ML & -0.2584 & 0.1661 & 0.2329 & 0.0666 & 0.0014 & 0.0058 & 0.0131 & 0.2111 & 0.2113 & 0.4500 \\
\hline & MPS & -0.3494 & 0.1139 & 0.2360 & 0.0605 & 0.0007 & 0.0044 & -0.1750 & 0.1760 & 0.2067 & 0.4471 \\
\hline & LS & -0.2992 & 0.1136 & 0.2031 & 0.0647 & 0.0012 & 0.0053 & -0.0063 & 0.2282 & 0.2283 & 0.4367 \\
\hline \multirow{3}{*}{$\mathrm{n}=300$} & ML & -0.3074 & 0.0620 & 0.1564 & 0.0569 & 0.0003 & 0.0035 & -0.0583 & 0.0812 & 0.0846 & 0.2446 \\
\hline & MPS & -0.3607 & 0.0488 & 0.1790 & 0.0559 & 0.0002 & 0.0033 & -0.1497 & 0.0731 & 0.0955 & 0.2778 \\
\hline & LS & -0.3172 & 0.0545 & 0.1552 & 0.0580 & 0.0003 & 0.0037 & -0.0618 & 0.1033 & 0.1071 & 0.2659 \\
\hline
\end{tabular}

$\alpha=2.0, \beta=1.5, \lambda=1.1$

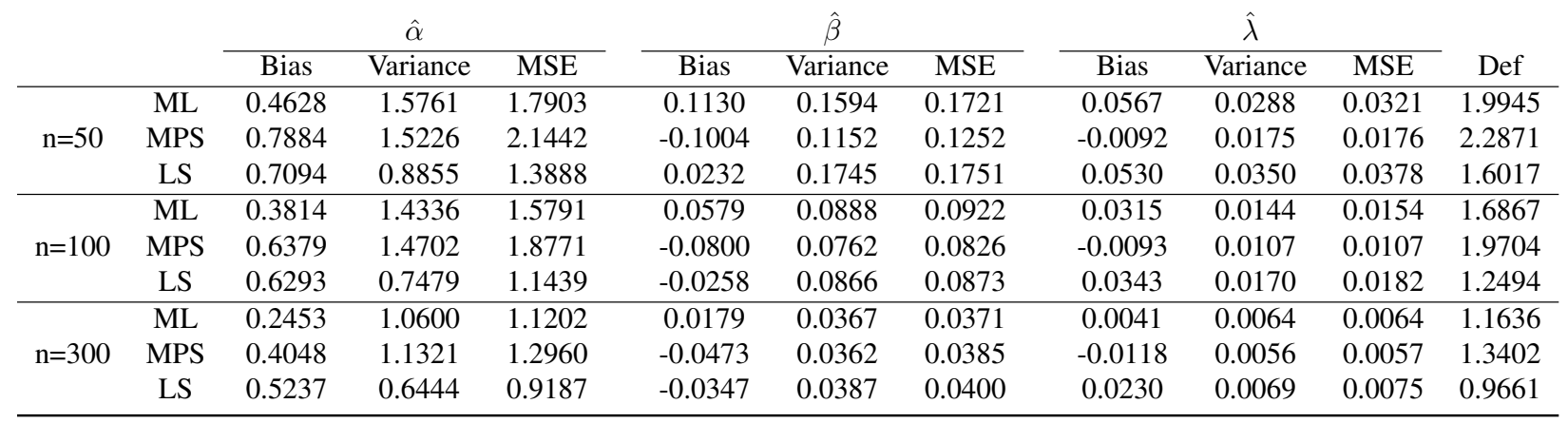




\section{Application}

In this section, an application to a real data set in which contains values of breaking stress of carbon fibers (in GPa) is provided. The data set, given in Table 2, is obtained from Nichols and Padgett (2006) who conducted a study on developing control chart when process measurements follow the Weibull distribution.

Table 2: The values of breaking stress of carbon fibers.

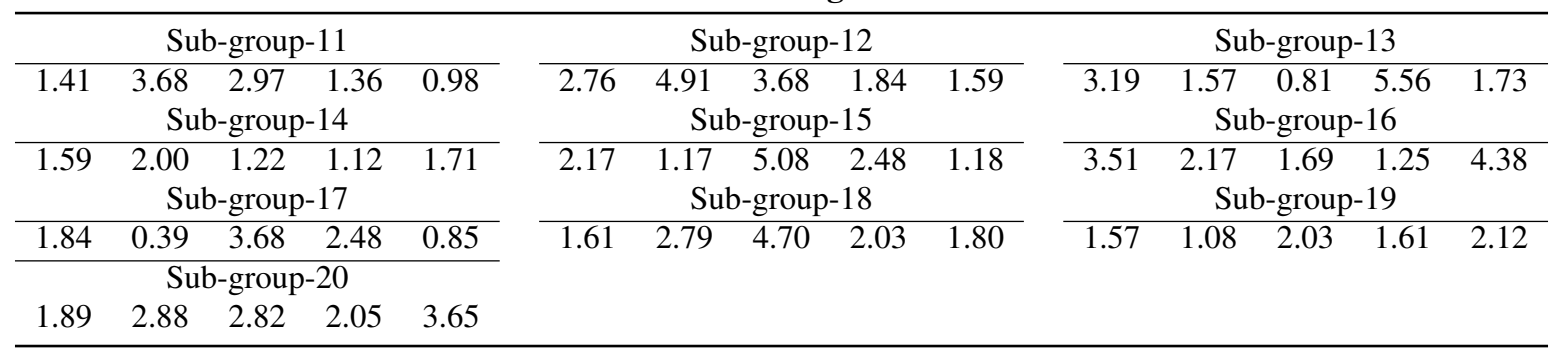

Recently, Jamal et al. (2019) modeled the breaking stress of carbon fibers data by using the Weibull, IKum, GIKw and GIKw-Weibull (GIKw-W) distributions. They showed that the GIKw-W distribution is preferable over the Weibull, IKum, and GIKw distributions in modeling the breaking stress of carbon fibers data when some goodness-of-fit measures are taken into account.

In this study, the APIK distribution is used for modeling the breaking stress of carbon fibres data; see Table 2. Beside, the GIKw-W and MOEIK distributions, which can be a strong alternative to the APIK distribution, are considered in the application. The IKum distribution is also included into the application to make comparisons complete. Modeling performances of the IKum, APIK, MOEIK, and GIKw-W distributions are compared by using well-known information criteria such as value of the $\ln L$, Akaike Information Criterion (AIC), corrected AIC (AICc), and goodness-of-fit measures the Kolmogorov-Smirnov (KS), root-mean-squared error (RMSE) and coefficent of determination $\left(R^{2}\right)$. As it is known, higher values of the $R^{2}$ and $\ln L$, and lower values of the AIC, AICc, KS, and RMSE indicate better fit.

The parameters $\alpha, \beta$, and $\lambda$ of the APIK distribution are estimated by using the ML, MPS, and LS methods as given in Section 4. For estimating the unknown parameters of the IKum, MOEIK, and GIKw-W distributions, the ML method is considered. The optimization tool "fminunc" available in software MATLAB2015b is utilized in estimating the unknown parameters of the IKum, APIK, MOEIK, and GIKw-W distributions. The corresponding results are given in Table 3. Also, values of the information criteria and goodness-of-fit measures for the IKum, APIK, MOEIK, and GIKw-W distributions are provided in Table 4. Furthermore, the pdf and cdf fitting plots of the IKum, APIK, MOEIK, and GIKw-W distributions are shown in Figure 2(a) and 2(b) for an illustration, respectively.

Table 3: The parameter estimates for the APIK, MOEIK, GIKw-W and IKUM distributions.

\begin{tabular}{ccccccc}
\hline & $\hat{a}$ & $\hat{b}$ & $\hat{\delta}$ & $\hat{\alpha}$ & $\hat{\beta}$ & $\hat{\lambda}$ \\
\cline { 2 - 7 } APIK $_{\mathrm{ML}}$ & - & - & - & 122.8373 & 9.0596 & 3.7420 \\
APIK $_{\mathrm{MPS}}$ & - & - & - & 122.8365 & 8.9634 & 3.7100 \\
APIK $_{\mathrm{LS}}$ & - & - & - & 122.8091 & 7.5129 & 3.5666 \\
\hline IKum & - & - & - & - & 17.9586 & 3.0450 \\
MOEIK & - & - & - & 4.6951 & 11.4699 & 15.4894 \\
GIKw-W & 0.0818 & 0.2161 & 6.6121 & 0.3117 & 2.7658 & - \\
\hline
\end{tabular}

Table 4: The values of information criteria and goodness-of-fit measures for the related distributions.

\begin{tabular}{|c|c|c|c|c|c|c|}
\hline \multirow[b]{3}{*}{$\mathrm{APIK}_{\mathrm{ML}}$} & \multicolumn{3}{|c|}{ Information criteria } & \multicolumn{3}{|c|}{ Goodness-of-fit measures } \\
\hline & $\ln L$ & AIC & AICc & $\mathrm{KS}$ & RMSE & $R^{2}$ \\
\hline & -73.9126 & 153.8252 & 154.3469 & 0.0761 & 0.0261 & 0.9919 \\
\hline $\mathrm{APIK}_{\mathrm{MPS}}$ & - & - & - & 0.0751 & 0.0264 & 0.9917 \\
\hline $\mathrm{APIK}_{\mathrm{LS}}$ & - & - & - & 0.0822 & 0.0250 & 0.9922 \\
\hline IKum & -75.9941 & 155.9882 & 156.2435 & 0.1121 & 0.0304 & 0.9874 \\
\hline MOEIK & -74.0793 & 154.1586 & 154.6803 & 0.0879 & 0.0338 & 0.9865 \\
\hline GIKw-W & -72.1572 & 154.3144 & 155.6780 & 0.0852 & 0.0264 & 0.9916 \\
\hline
\end{tabular}




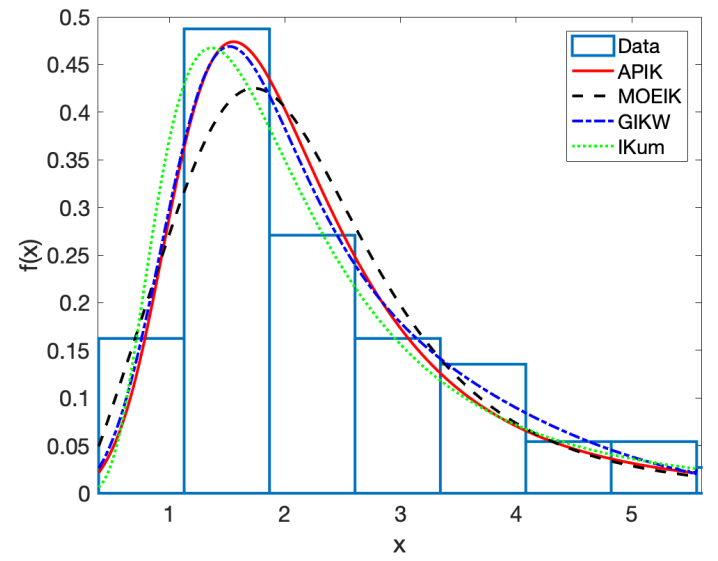

(a) pdfs fitting plot

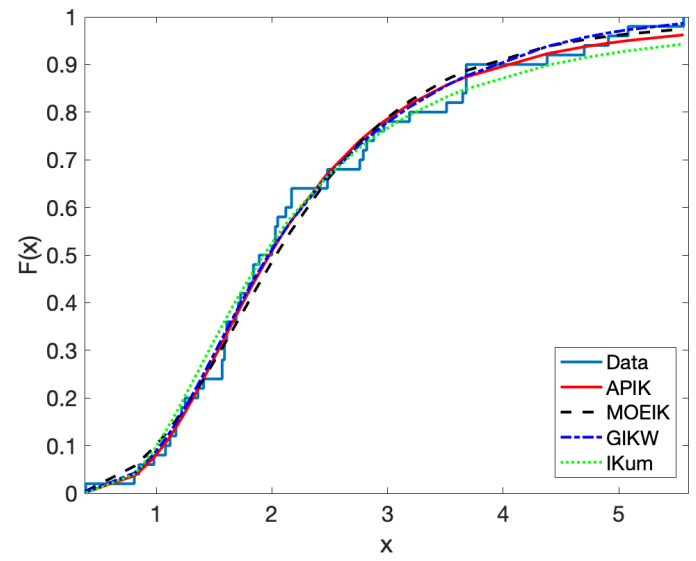

(b) cdfs fitting plot

Figure 2: Fitting plots for pdfs (a) and cdfs (b) of the APIK distributions for the ML estimates of the parameters. Concerning the goodness-of-fit measures for the APIK distribution calculated by using the ML, MPS, and LS estimates of the corresponding parameters, it is seen that the ML, MPS, and LS methods give more or less the same $R^{2}$ values. The KS and RMSE values resulted in the ML and MPS methods are close to each others.

In comparisons for the modeling performances of the APIK, MOEIK, GIKw-W, and IKum distributions, it is seen that the APIK distribution has the smallest AIC and AICc values. Note that the APIK and MOEIK distributions have three parameters in the meanwhile the GIKw-W distribution has five parameters.

When the goodness-of-fit measures are taken into account to compare the modeling performances of the APIK, MOEIK, GIKw-W, and IKum distributions, the APIK distribution has the smallest KS and RMSE values. Also, the GIKw-W distribution comes after the APIK distribution, since they have more or less the same RMSE and $R^{2}$ values.

Overall, in terms of the information criteria AIC and AICc, the APIK distribution is preferable over the IKum, MOEIK, and GIKw-W distributions. Also, the APIK distribution shows better modeling performance than the IKum, MOEIK, and GIKw-W distributions when the KS criterion is considered. Although the APIK distribution has fewer number of parameters than the GIKw-W distribution, it stands ahead of the GIKw-W distribution in modeling the breaking stress of carbon fibres data. To sum up, it is shown that the APIK distribution can be an alternative to the GIKw-W and MOEIK distributions, since it stands out in much more criteria.

\section{Conclusion}

In this study, the APIK distribution is obtained by using the APT method. Then, the sub-models and related distributions of the APIK distribution are obtained as well. To the best of the Authors' knowledge, some of its sub-models obtained in this study have not been introduced yet. The parameters of the APIK distribution are estimated by using the ML, MPS, and LS methods. The Monte-Carlo simulation study is conducted to show efficiencies of the considered estimation methods in estimating the parameters $\alpha, \beta$, and $\lambda$ of the APIK distribution. The real data set including values of breaking stress of carbon fibers is used to show the modeling capability of the APIK distribution. Also, the MOEIK and GIKw-W distributions, which can be considered strong alternatives to the APIK, are included in the application to make the study complete. As it can be seen from Table 4 that the APIK distribution has better modeling performance when compared with its rivals in many criteria. Overall, based on the results obtained in this study, the Authors hope that the APIK distribution can be useful for engineering studies as well as many other fields.

\section{Acknowledgements}

The Authors would like to thank the Reviewers for their valuable comments and suggestions. 


\section{References}

1. Abu-Moussa, M. and El-Din, M. (2018). On estimation and prediction for the inverted Kumaraswamy distribution based on general progressive censored samples. Pakistan Journal of Statistics and Operation Research, 14:717-736, https://doi.org/10.18187/pjsor.v14i3.2103.

2. Akgül, F., Şenoğlu, B., and Arslan, T. (2016). An alternative distribution to Weibull for modeling the wind speed data: Inverse Weibull distribution. Energy Conversion and Management, 114:234-240, https://doi.org/10.1016/j.enconman.2016.02.026.

3. AL-Fattah, A., El-Helbawy, A., and Al-Dayian, G. (2017). Inverted Kumaraswamy distribution: Properties and estimation. Pakistan Journal of Statistics, 33:37-61.

4. Aldahlan, M. (2020). Alpha power transformed Log-Logistic distribution with application to breaking stress data. Advances in Mathematical Physics, Article ID 2193787:1-9, https://doi.org/10.1155/2020/2193787.

5. Almongy, H., Almetwally, E., and Mubarak, A. (2021). Marshall-Olkin alpha power Lomax distribution: Estimation methods, applications on Physics and Economics. Pakistan Journal of Statistics and Operational Research, 17:137-153, http://dx.doi.org/10.18187/pjsor.v17i1.3402.

6. Aly, H. and Abuelamayem, O. (2020). Multivariate inverted Kumaraswamy distribution: Derivation and estimation. Mathematical Problems in Engineering, Article ID 6349523:1-27, https://doi.org/10.1155/2020/6349523.

7. Arslan, G. and Oncel, S. (2017). Parameter estimation of some Kumaraswamy-G type distributions. Mathematical Sciences, 11:131-138, https://doi.org/10.1007/s40096-017-0218-0.

8. Arslan, T., Acitas, S., and Şenoğlu, B. (2017). Generalized lindley and power Lindley distributions for modeling the wind speed data. Energy Conversion and Management, 152:300-311, https://doi.org/10.1016/j.enconman.2017.08.017.

9. Bagci, K., Arslan, T., and Celik, H. (2021). Inverted Kumaraswamy distribution for modeling the wind speed data: Lake van, turkey. Renewable and Sustainable Energy Reviews, 135:110110, https://doi.org/10.1016/j.rser.2020.110110.

10. Bagci, K., Erdogan, N., Arslan, T., and Celik, H. (2019). Alpha power inverted Kumaraswamy distribution: Properties and application. In International Conference on Computational Mathematics and Engineering Sciences (CMES-2019), page 54, Antalya, Turkey.

11. Barreto-Souza, W. and Simas, A. (2014). The exp-G family of probability distributions. Brazilian Journal of Probability and Statistics, 27:84-109, https://www.jstor.org/stable/43601236.

12. Basheer, A. (2019). Alpha power inverse Weibull distribution with reliability application. Journal of Taibah University for Science, 13:423-432, https://doi.org/10.1080/16583655.2019.1588488.

13. Biçer, H. (2019). Properties and inference for a new class of generalized Rayleigh distributions with an application. Open Mathematics, 17:700-715, https://doi.org/10.1515/math-2019-0057.

14. Bulut, Y., Doğru, F., and Arslan, O. (2021). Alpha power Lomax distribution: Properties and application. Journal of Reliability and Statistical Studies, 14:17-32, https://doi.org/10.13052/jrss0974-8024.1412.

15. Dey, S., Alzaatreh, A., Zhang, C., and Kumar, D. (2017). A new extension of generalized Exponential distribution with application to ozone data. Ozone: Science \& Engineering, 39:273-285, https://doi.org/10.1080/01919512.2017.1308817.

16. Dey, S., Gosh, I., and Kumar, D. (2019a). Alpha power transformed Lindley distribution: Properties and associated inference with application to earthquake data. Annals of Data Science, 6:623-650, https://doi.org/10.1007/s40745-018-0163-2.

17. Dey, S., Nassar, M., and Kumar, D. (2019b). Alpha power transformed inverse Lindley distribution: A distribution with an upside-down bathtub-shaped hazard function. Journal of Computational and Applied Mathematics, 385:130-145, https://doi.org/10.1016/j.cam.2018.03.037.

18. Erdogan, N., Bagci, K., Arslan, T., and Celik, H. (2021). Alpha power Maxwell distribution: Properties and application. Journal of Mathematical Modeling, 9:585-598, https://doi.org/10.22124/JMM.2021.17987.1553.

19. Hameed, B., Salman, A., and Kalaf, B. (2020). On the estimation of $P\left(Y_{1}<X<Y_{2}\right)$ in case inverted Kumaraswamy distribution. Iraqi Journal of Science, 61:845-853, https://doi.org/10.24996/ijs.2020.61.4.18.

20. Hassan, A., Elgarhy, M., Mohamd, R., and Alrajhi, S. (2019). On the alpha power transformed power Lindley distribution. Journal of Probability and Statistics, 8024769:1-19, https://doi.org/10.1155/2019/8024769.

21. Hassan, A., Mohamd, R., Elgarhy, M., and Fayomi, A. (2018). Alpha power transformed extended exponential distribution: properties and applications. Journal of Nonlinear Sciences and Applications, 12:239-251, 
http://dx.doi.org/10.22436/jnsa.012.04.05.

22. Ihtisham, S., Khalil, A., Manzoor, S., Khan, S., and Ali, A. (2019). Alpha-power Pareto distribution:Its properties and applications. PLoS ONE, 14:1-15, https://doi.org/10.1371/journal.pone.0218027.

23. Iqbal, Z., Tahir, M., Riaz, N., Ali, S., and Ahmad, M. (2017). Generalized inverted Kumaraswamy distribution: Properties and application. Open Journal of Statistics, 7:645-662, https://doi.org/10.4236/ojs.2017.74045.

24. Jamal, F., Nasir, M., Ozel, G., Elgarhy, M., and Khan, N. (2019). Generalized inverted Kumaraswamy generated family of distributions: Theory and applications. Journal of Applied Statistics, 46:356-365, https://doi.org/10.1080/02664763.2019.1623867.

25. Jones, M. (2009). Kumaraswamy's distribution: A beta-type distribution with some tractability advantages. Statistical Methodology, 6:70-81, https://doi.org/10.1016/j.stamet.2008.04.001.

26. Jones, M. (2018). Letter to the editor concerning "a new method for generating distributions with an application to exponential distribution" and "alpha power weibull distribution: Properties and applications". Communications in Statistics-Theory and Methods, 47:5096, https://doi.org/10.1080/03610926.2017.1386314.

27. Kumaraswamy, P. (1980). A generalized probability density function for double-bounded random processes. Journal of Hydrology, 46:79-88, https://doi.org/10.1016/0022-1694(80)90036-0.

28. Lee, C., Famoye, F., and Alzaatreh, A. (2013). Methods for generating families of univariate continuous distributions in the recent decades. Wiley Interdisciplinary Reviews: Computational Statistics, 5:219-238, https://doi.org/10.1002/wics.1255.

29. Mahdavi, A. and Kundu, D. (2017). A new method for generating distributions with an application to Exponential distribution. Communications in Statistics-Theory and Methods, 46:6543-6557, https://doi.org/10.1080/03610926.2015.1130839.

30. Mead, M., Cordeiro, G., Afify, A., and Al-Mofleh, H. (2019). The alpha power transformation family: Properties and applications. Pakistan Journal of Statistics and Operational Research, 15:525-545, https://doi.org/10.18187/pjsor.v15i3.2969.

31. Nadarajah, S., Nassiri, V., and Mohammadpour, A. (2014). Truncated-exponential skew-symmetric distributions. Statistics, 48:872-895, https://doi.org/10.1080/02331888.2013.821474.

32. Nassar, M., Afify, A., and Shakhatreh, M. (2020). Estimation methods of alpha power exponential distribution with applications to engineering and medical data. Pakistan Journal of Statistics and Operational Research, 16:149-166, https://doi.org/10.18187/pjsor.v16i1.3129.

33. Nassar, M., Alzaatreh, A., Mead, M., and Abo-Kasem, O. (2017). Alpha power Weibull distribution: Properties and applications. Communications in Statistics-Theory and Methods, 46:10236-10252, https://doi.org/10.1080/03610926.2016.1231816.

34. Nichols, M. and Padgett, W. (2006). A bootstrap control chart for Weibull percentiles. Quality and Reliability Engineering International, 22:141-151, https://doi.org/10.1002/qre.691.

35. Ramadan, D. and Magdy, A. (2018). On the alpha-power inverse Weibull distribution. International Journal of Computer Applications, 11:6-12, https://doi.org/10.5120/ijca2018917657.

36. Reyad, H., Jamal, F., Othman, S., and Yahia, N. (2019). The Topp-Leone generalized inverted Kumaraswamy distribution: Properties and applications. Asian Research Journal of Mathematics, 13:1-15, https://doi.org/10.9734/arjom/2019/v13i330107.

37. Sherwani, R., Waqas, M., Saeed, N., Farooq, M., Raza, M., and Jamal, F. (2021). Transmuted inverted Kumaraswamy distribution: Theory and applications. Punjab University Journal of Mathematics, 53:29-45.

38. Swain, J., Venkatraman, S., and Wilson, J. (1988). Least-squares estimation of distribution functions in Jhonson's translation system. Journal of Statistical Computation and Simulation, 24:271-297, https://doi.org/10.1080/00949658808811068.

39. Unal, C., Cakmakyapan, S., and Ozel, G. (2018). Alpha power inverted exponential distribution: Properties and application. Gazi Journal of Science, 31:957-965.

40. Usman, R. and ul Haq, M. (2020). The Marshall-Olkin extended inverted Kumaraswamy distribution: Theory and applications. Journal of King Saud University, 32:356-365, https://doi.org/10.1016/j.jksus.2018.05.021.

41. Volovskiy, G. and Kamps, U. (2020). Maximum product of spacings prediction of future record values. Metrika, 83:853-868, https://doi.org/10.1007/s00184-020-00767-1. 